\title{
Sustitución de los Agregados por Escoria de Cobre en la Elaboración del Concreto
}

RESPONSABLE: Ing. Gualberto Tejada Bedoya

\section{RESUMEN. La finalidad de este artículo es el estudio de la viabilidad técnica del uso de las escorias de cobre como parte integrante del concreto. Se orienta a la utilización de la escoria de cobre como agregado para concreto, en el cual se reemplazó escoria por arena en porcentajes de 10, 20 y $30 \%$ con relación al volumen de la arena.}

La variable dependiente es la resistencia a la compresión, este fue el parámetro para la elección de los porcentajes óptimos a utilizarse; además de la comparación de los concretos con escoria, concretos convencionales preparados con la misma dosificación.

En lo que se refiere a su uso como agregado, el concreto de mejores características y resistencias más elevadas hasta en un $62 \%$ a edades tempranas en comparación con un concreto convencional, fue el preparado con $20 \%$ escoria y $80 \%$ de arena.
MIEMBros: Ing. Zenon Sarmiento Mejia

Ing. Carlos Huisa Ccori

\begin{abstract}
The purpose is to study the technical feasibility of using copper slag as part of the concrete. It is oriented to the study of copper slag as aggregate for Specifically, the slag which was replaced by sand in percentages of 10,20 and $30 \%$ compared to the volume of sand.
\end{abstract}

The dependent variable is the resistance to compression, this was the parameter for choosing the optimal rates used; addition to comparing the slag concrete, conventional concrete prepared with the same dosage.

In regard to its use as aggregate, concrete, better features and higher resistance up to $62 \%$ at early ages in relation to a particular conventional, was prepared with $20 \%$ slag and $80 \%$ sand.

\section{MATERIALY MÉTODO}

Es conocido, en el medio técnico, que la ingeniería se revela como el sector con el más alto potencial para el uso de residuos, principalmente por el gran volumen de materia prima que consume, independiente del área.

Estudios desarrollados por OCDE (Organitation for Economic Cooperation and Development) y RILEM (Reunion International de Laboratorios de Essais Matériaux) establecieron una propuesta de criterio general de evaluación del residuo para el uso en construcción civil (CINCOTTO, 1988):

a) La cantidad de residuo disponible debe ser suficientemente grande para justificar el desarrollo de sistemas de procesado y transporte;

b) La distancia de transporte que involucra debe ser compatible con el uso de los materiales convencionales; $y$

c) El material no debe ser potencialmente nocivo durante la construcción o después de su incorporación al producto final.

La OCDE propone una clasificación de los residuos en cinco grupos que se usan internacionalmente (CINCOTTO, 1988):

Grupo 1-Residuos de los derivado mineros;
Grupo 2 - Residuos de los derivados metalúrgicos;

Grupo 3 - Residuos industriales;

Grupo 4-Residuos municipales; $y$

Grupo 5 -Residuos agrícolas y forestales.

De acuerdo con estos criterios y verificadas las compatibilidades técnicas, los materiales podrían distribuirse en cuatro clases de acuerdo con la potencialidad de su aplicación en ingenieria civil (CINCOTTO, 1988):

- Clase1-Materiales con el potencial de aplicación máxima;

Clase2-Materiales con el potencial de la buena aplicación, pero inferior al de la clase 1 ;

Clase3-Materiales con bajo potencial de la aplicación;y

Clase4-Materiales con muy poco potencial de aplicación.

A través de la revisión bibliográfica cumplida, se verificó que son muy pocos los trabajos publicados con respecto al uso de escoria cobriza. La información que uno tiene es que prácticamente de cada volumen de escoria generada que se deposita en los patios de los generadores de metal, una cantidad pequeña se ha usado como abrasivo en algunos países como Singapur y Tailandia (PAN ABRASIVES, 2000). También en Chile, que es el segundo productor de cobre del mundo, 
la escoria se ha depositado a cielo abierto y un volumen pequeño está siendo comercializado como abrasivo (TECNOLOGICALCENTER, 2000).

\section{Caracterización de la Escoria de Cobre}

\section{Recolección de la Muestra}

Para logra una muestra global representativa de la escoria desechada, ha sido necesario constituirse en la fundición de Ilo que se ubica aproximadamente a 16.1 $\mathrm{km}$ al norte de la ciudad portuaria de Ilo y se puede llegar a ella por carretera.

La escoria se encuentra acumulada en una cantera artificial, la cual está ubicada a orillas del mar.

La escoria, naturalmente después del proceso de enfriamiento, se encuentra entre un tamaño máximo de 2 " hasta aproximadamente la malla $\mathrm{N}^{\circ} 16 \mathrm{ASTM}$, por lo cual fue necesario tamizar el material, para lo cual se usó la malla $1 / 2$ ", tomándose una muestra representativa de $50 \mathrm{~kg}$ para la realización de los ensayos como agregado.

\section{Composición Química.}

La composición química de la escoria es la siguiente:

\begin{tabular}{|c|c|c|}
\hline \multirow{2}{*}{ ELEMENTOS / } & \multicolumn{2}{|c|}{ CONTENIDO } \\
\cline { 2 - 3 } COMPUESTOS & $\%$ & Oz / Tonelada \\
\hline $\mathrm{Cu}$ & 10,68 & - \\
$\mathrm{Fe}$ & 42,40 & - \\
$\mathrm{Fe} 3 \mathrm{O} 4$ & 19,70 & - \\
$\mathrm{SiO} 2$ & 19,74 & - \\
$\mathrm{Al} 2 \mathrm{O} 3$ & 5,04 & - \\
$\mathrm{CaO}$ & 1,38 & - \\
$\mathrm{CuO}$ & 0,25 & - \\
$\mathrm{Mo}$ & 0,06 & - \\
$\mathrm{S}$ & 3,52 & - \\
$\mathrm{Ag}$ & - & 0,81 \\
$\mathrm{Au}$ & - & $\mathrm{Tr}$ \\
\hline
\end{tabular}

Composición química de la escoria.

\section{RESULTADOS}

\begin{tabular}{|l|c|c|cc|c|}
\hline \multicolumn{1}{|c|}{ DESCRIPCION } & UNIDAD & CEMENTO & AGR FINO & $\begin{array}{c}\text { AGR. } \\
\text { GRUESO }\end{array}$ & ESCORIA \\
\hline Abrasión & $\%$ & & & & 16.9 \\
Peso Especifico & Gricm 3 & 2.84 & 2.586 & 2.571 & 3.367 \\
\hline Peso Unitario Varilado & $\mathrm{Kg} / \mathrm{m} 3$ & & & 1589 & \\
Peso Unitario Suelto & $\mathrm{Kg} / \mathrm{m} 3$ & 1500 & 1494 & 1431 & 1880 \\
\hline Humedad Natura & $\%$ & & 1.2 & 0.66 & 0.39 \\
\hline Absorción & $\%$ & & 0.47 & 1.34 & 0.56 \\
\hline Móduo de Fineza & & & 2.91 & & \\
\hline Tamario Maximo & polg & & & 1 & \\
\hline
\end{tabular}

Resumen de Propiedades Química de la Escoria en Compa Ración con un Agregado Pétreo.

\begin{tabular}{|l|c|c|c|}
\hline \multicolumn{1}{|c|}{ DESCRIPCION } & UNIDAD & ESCORIA & AGR.PETREO \\
\hline Sulfatos & ppm & Trazas & 344 \\
\hline Cloruros & ppm & 419 & 96.5 \\
Sólidos Totales & $\mathrm{ppm}$ & 1800 & 726 \\
\hline
\end{tabular}

Consideraciones sobre Posibles Aplicaciones de la Escoria a Partir de sus Características.

La escoria del alto-horno puede tener varias aplicaciones como materia prima para la producción del cemento, como adición en la ejecución del concreto, como agregado para la producción de concreto, premoldeados y pavimentación, como agregado leve en la producción de concreto y la escoria para los aislamientos térmicos (KRUGER, 1995).

\section{DISCUSIÓN}

\section{Uso como Aditivo en la Producción del Cemento.}

A Loriet se le atribuye la primera referencia del valor hidráulico de una escoria, en 1774. En 1865 fue lanzado en Alemania un ligante hidráulico para una mezcla de cal y espuma. También en Alemania, en 1882, Prussing hizo posible la producción industrial del cemento Portland adicionado con escoria. Sin embargo, entre 1880 y 1890 las propiedades de este nuevo producto son más conocidas. Sin embargo, encontraron mucha resistencia en el medio técnico y por parte de los fabricantes de cemento (COUTINHO, 1997). La reactividad de la escoria del alto-horno es la propiedad principal que hace posible su uso como aditivo. La reactividad depende de la composición química, el valor de la fase vítrea y de la finura.

\section{Uso como Agregado}

Las escorias del alto-horno pueden ser molidas y graduadas para el uso como agregado fino o partidas para ser usadas como agregado grueso. Las escorias más usadas para este fin son las de enfriamiento lento.

En Francia, la escoria del alto-horno se usa como agregado para enriquecimiento de las capas de base de pavimentos de carreteras y autopistas. El agregado natural es usado, de $5 \%$ a $20 \%$ de escoria granulada y $1 \%$ de cal (VENUAT, mencionado por LOUZADA, 1991)

\begin{tabular}{|c|c|c|c|c|}
\hline \multirow[t]{2}{*}{ MATERIALES } & \multirow{2}{*}{$\begin{array}{c}\text { Co Refer. } \\
0 \%\end{array}$} & \multicolumn{3}{|c|}{$\begin{array}{l}\mathrm{C}^{\circ} \text { con \% de Agregado de } \\
\text { escoria }\end{array}$} \\
\hline & & $10 \%$ & $20 \%$ & $30 \%$ \\
\hline $\begin{array}{l}\text { Cemento } \\
\left(\mathrm{Kg} / \mathrm{m}^{3}\right)\end{array}$ & 346,02 & 346,02 & 346,02 & 346,02 \\
\hline $\begin{array}{l}\text { Agua de } \\
\text { Diseño }\left(1 \mathrm{t} / \mathrm{m}^{3}\right) \\
\text { Agregado } \\
\text { Grueso }\left(\mathrm{Kg} / \mathrm{m}^{3}\right)\end{array}$ & $\begin{array}{r}197,87 \\
1102,85\end{array}$ & $\begin{array}{l}200,86 \\
921,62\end{array}$ & $\begin{array}{c}214,7 \\
985,18\end{array}$ & 221,62 \\
\hline $\begin{array}{l}\text { Agregado fino } \\
\left(\mathrm{Kg} / \mathrm{m}^{3}\right)\end{array}$ & 683,22 & 708,56 & 550,82 & 447,38 \\
\hline Escoria $\left(\mathrm{kg} / \mathrm{m}^{3}\right)$ & & 102,69 & 178,45 & 249,16 \\
\hline
\end{tabular}

Diseños de mezcla por $m^{3}$ para los diferentes valores de agregado. 


\section{Evaluación del Concreto}

\begin{tabular}{|c|c|c|c|c|c|}
\hline Combinaciones & Agr. FinotmB & Escoriam3 & $a d c$ & $\begin{array}{l}\text { Slump } \\
\text { pulg }\end{array}$ & $\begin{array}{c}\text { Resistencia a } \\
28 \text { dias }\end{array}$ \\
\hline \%\%Referencia & 683.22 & 0 & 057 & 2 & 226 \\
\hline $10 \%$ escoria & 708.56 & 10269 & 0.58 & 2 & 296 \\
\hline $20 \%$ escoria & 550.82 & 178.45 & $0 \approx 2$ & $2 \frac{1}{2}$ & 404 \\
\hline $30 \%$ escoria & 447.38 & 249.16 & 064 & $21 / 2$ & 313 \\
\hline
\end{tabular}

Cuadro consolidado de concretos con agregado de escoria.

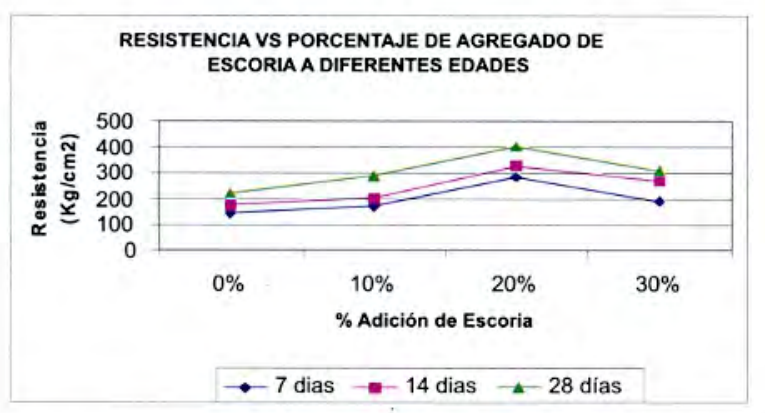

Evolución de la resistencia a la comprensión para los diferentes porcentajes de agregado de escoria de cobre.

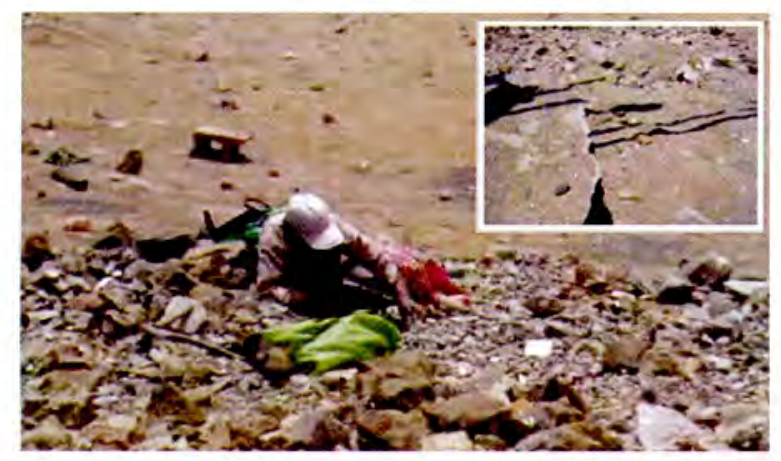

Recolección manual de la escoria de cobre.

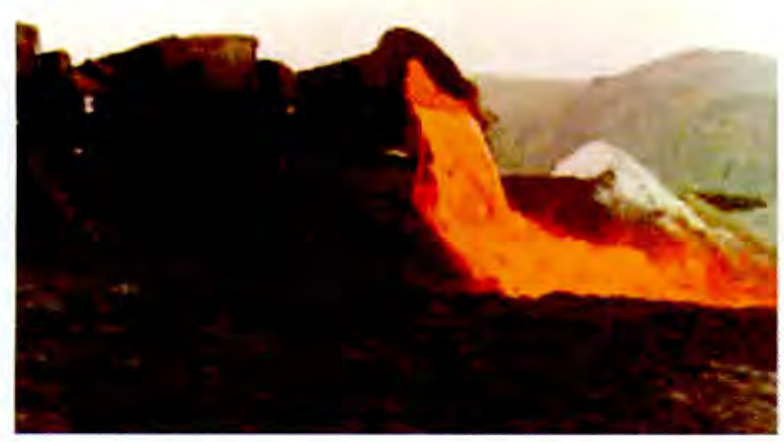

Deposición de la escoria en la superficie.

\section{CONCLUSIONES}

1. El porcentaje óptimo para la sustitución de agregado de escoria por agregado fino en volumen, según la investigación realizada, es $20 \%$ escoria - $80 \%$ Agregado fino. Porque la resistencia es mayor.

2. Al adquirir resistencias superiores a las de diseño, para el caso de la investigación $210 \mathrm{~kg} / \mathrm{cm} 2$, se podría tener una reducción de los materiales del concreto puesto que con menos cantidades se obtendrían iguales resistencias altas, en comparación con un concreto convencional

3. La utilización de la escoria como adición y agregado ayudaría al reciclaje de la escoria granulada que se encuentra en las playas de la Fundición de Ilo. Además reducirían los Impactos Ambientales que se producen en la Fauna Marina.

\section{REFERENCIAS BIBLIOGRÁFICAS}

INSTITUTO MEXICANO DEL CEMENTO Y DEL CONCRETO, Tecnología del concreto, Tomo I, II y III, Editorial Limusa Noriega Editores, Mexico, 1988.

ACI, CAPÍTULO PERUANO, "Tecnología del concreto", Primer Congreso Nacional de Ingeniería Civil y Construcción, Martegraf E.I.R.L, Lima, 1998, Capítulo I Enrique Rivva Lopez, Capitulo II Juan Harman Infantes.

ACI, CAPITULO PERUANO, Supervisión de obras de concreto, Tercera edición, Editorial WH Editores SRLtda, Lima, 1995. Capítulo I Enrique Pasquel Carvajal, Capítulo II Ana Biondi Saw.

CEF, COMISIÓN FEDERAL DE ELECTRICIDAD, Manual de tecnología de concreto, Instituto de Ingeniería, UNAM, Sección I Definición de requisitos de los componentes del concreto fresco. Sección II Características y propiedades del concreto fresco. Editoria Limusa Noriega Editores, México, 1994.

Enrique RIVVA LÓPEZ, "Tecnología del concreto", Diseño de Mezclas, Peru 1992.

ACI - PERU, Enrique RIVVA LÓPEZ, "Naturaleza y Materiales del Concreto", II Congreso Nacional de Estructuras y Construccion, Primera Edición, Editorial Capítulo Peruano ACI, San Isidro, Lima, 2000.

AMARAL DE LIMA, Luciana. Hormigones con Escoria de Alto Horno, Universidad Politécnica de Catalunya Escuela Técnica Superior de Ingeniería de Caminos Canales y Puertos". Tesis Doctoral, Barcelona, noviembre, 1999. 
ANEXO

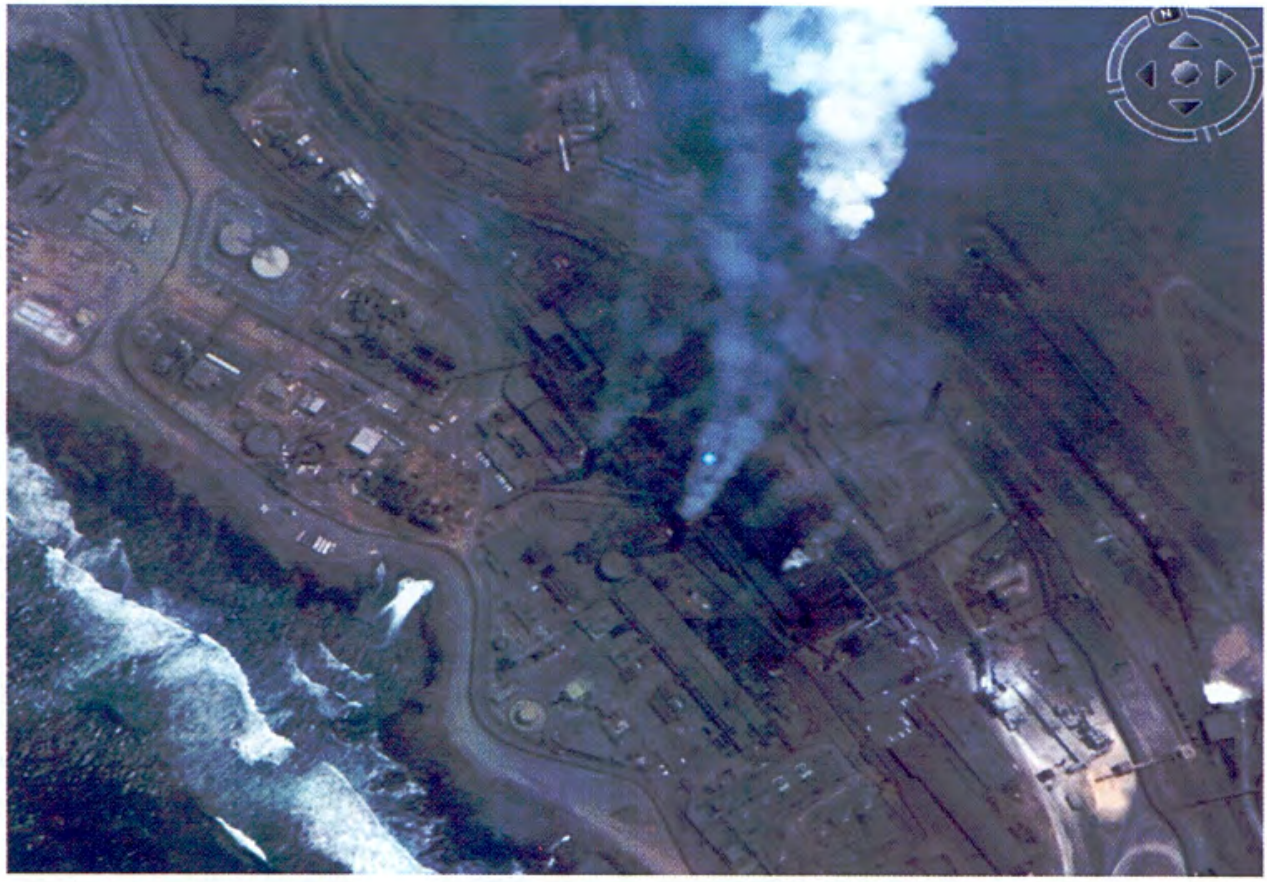

Foto: La playa y los hornos que producen la escoria de Cobre

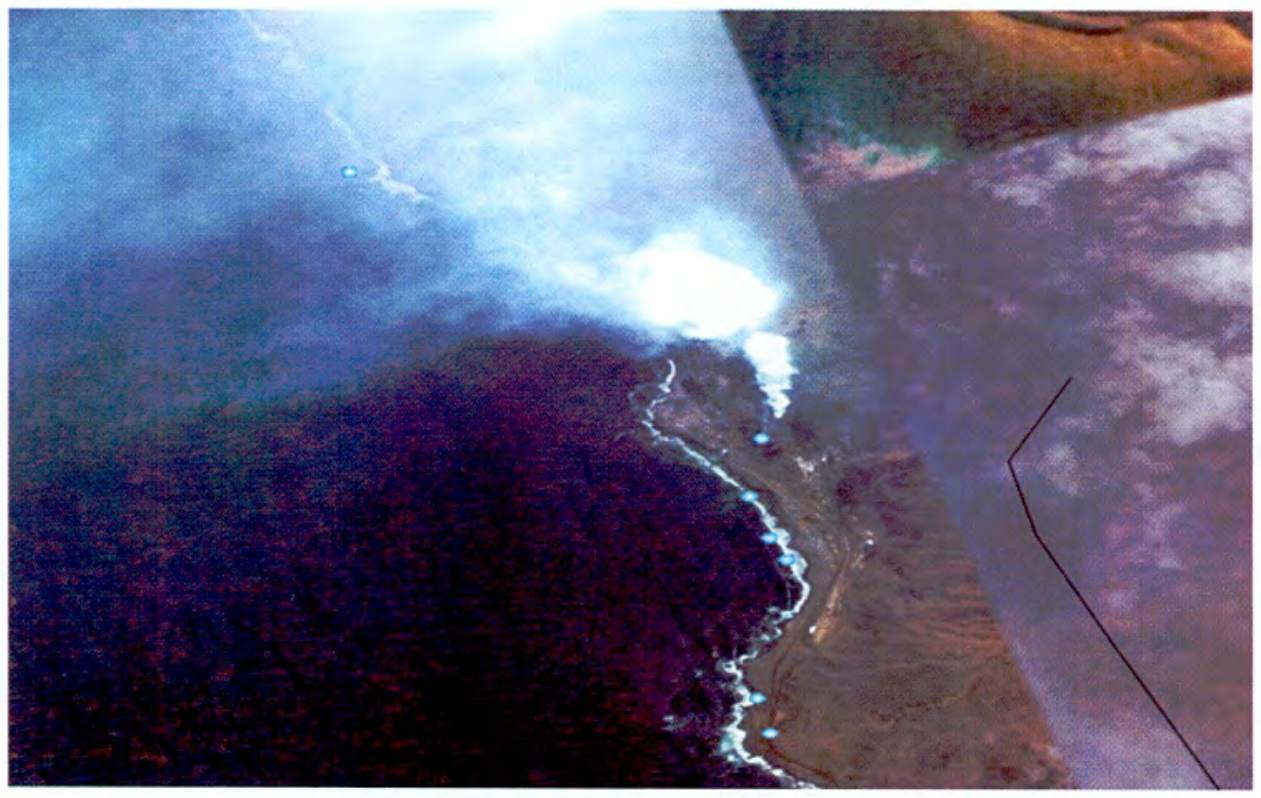

Foto: Ubicación de la planta de fundición Ilo frente a lecho marino 